\title{
Bandwidth Enhancement and Further Size Reduction of a Class of Miniaturized Slot Antennas
}

\author{
Nader Behdad, Student Member, IEEE, and Kamal Sarabandi, Fellow, IEEE
}

\begin{abstract}
In this paper, new methods for further reducing the size and/or increasing the bandwidth (BW) of a class of miniaturized slot antennas are presented. This paper examines techniques such as parasitic coupling and inductive loading to achieve higher BW and further size reduction for this class of miniaturized slot antennas. The overall $\mathrm{BW}$ of a proposed double resonant antenna is shown to be increased by more than 94\% compared with a single resonant antenna occupying the same area. The behavior of miniaturized slot antennas, loaded with series inductive elements along the radiating section is also examined. The inductive loads are constructed by two balanced short circuited slot lines placed on opposite sides of the radiating slot. These inductive loads can considerably reduce the antenna size at its resonance. Prototypes of a double resonant antenna at $850 \mathrm{MHz}$ and inductively loaded miniaturized antennas at around $1 \mathrm{GHz}$ are designed and tested. Finally the application of both methods in a dual band miniaturized antenna is presented. In all cases measured and simulated results show excellent agreement.
\end{abstract}

Index Terms-Slot antennas, electrically small antennas, parasitic antennas, multifrequency antennas.

\section{INTRODUCTION}

C URRENT advancements in communication technology and significant growth in the wireless communication market and consumer demands demonstrate the need for smaller, more reliable and power efficient, integrated wireless systems. Integrating entire transceivers on a single chip is the vision for future wireless systems. This has the benefit of cost reduction and improving system reliability. Antennas are considered to be the largest components of integrated wireless systems; therefore antenna miniaturization is a necessary task in achieving an optimal design for integrated wireless systems. The subject of antenna miniaturization is not new and has been extensively studied by various authors [1]-[4]. Early studies have shown that for a resonant antenna, as size decreases, bandwidth (BW) and efficiency will also decrease [1]. This is a fundamental limitation which, in general, holds true independent of antenna architecture. However, research on the design of antenna topologies and architectures must be carried out to achieve maximum possible $\mathrm{BW}$ and efficiency for a given antenna size. Impedance matching for small antennas is also challenging and often requires external matching networks; Therefore antenna topologies and structures which inherently

\footnotetext{
Manuscript received May 22, 2003; revised September 30, 2003. This work was supported in part by the Engineering Research Centers program of the National Science Foundation (NSF) under Award EEC-9986866 and by the U.S. Army Research Office under Contract DAA-99-1-01971.

The authors are with The Radiation Laboratory, Department of Electrical Engineering and Computer Science, The University of Michigan, Ann Arbor, MI 48109-2122 USA (e-mail: behdad@engin.umich.edu).

Digital Object Identifier 10.1109/TAP.2004.832330
}

allow for impedance matching are highly desirable. The fundamental limitation introduced by Chu [1] and later re-examined by McLean [4] relates the radiation $\mathrm{Q}$ of a single resonance antenna with its BW. However, whether such limitation can be directly extended to multiresonance antenna structures or not is unclear. In fact, through a comparison with filter theory, designing a relatively wideband antenna may be possible using multipole (multiresonance) high Q structures. In this paper we examine the applicability of multiresonance antenna structures to enhance the BW of miniaturized slot antennas.

Different techniques have been used for antenna miniaturization such as: miniaturization using optimal antenna topologies [5]-[7] and miniaturization using magneto-dielectric materials [8], [9]. In pursuit of antenna miniaturization while maintaining ease of impedance matching and attaining relatively high efficiency, a novel miniaturized slot antenna was recently presented [6]. Afterwards, a similar architecture in the form of a folded antenna geometry was presented in order to increase the BW of the previously mentioned miniaturized slot antenna [7]. Here we re-examine this topology [6] and propose modifications that can result in further size reduction or BW enhancement without imposing any significant constraint on impedance matching or cross polarization level. In Section II, a dual-resonant antenna topology is examined for BW enhancement. This miniaturized antenna shows a BW which is 94\% larger than that of a single-resonant miniaturized antenna with the same size.

Using series inductive elements distributed along the antenna aperture results in the increase of inductance per unit length of the line. Therefore the guided wavelength of the resonant slot line is shortened. Thus, the overall length of the antenna is decreased. In Section III, this technique is first demonstrated using a standard resonant slot antenna and then incorporated in the miniaturized antenna topology of [6] to further reduce the resonant frequency without increasing the area occupied by the antenna.

The aforementioned techniques for BW enhancement and further size reduction can be used individually or in combination. The combined application of the techniques of Sections II and III is presented in Section IV by demonstrating the design of a dual band miniaturized slot antenna.

\section{Miniaturized Slot Antenna With Enhanced BW}

\section{A. Design Procedure}

In this section the design of coupled miniaturized slot antennas for BW enhancement is studied. The configuration of the proposed coupled slot antenna is shown in Fig. 1(b) where 


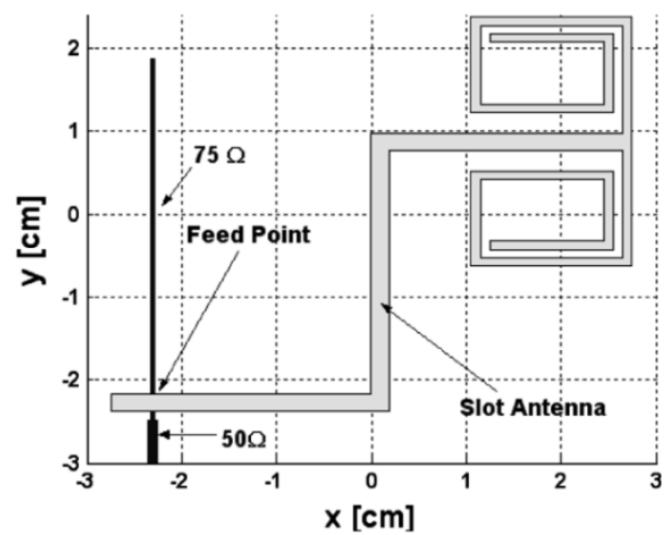

(a)

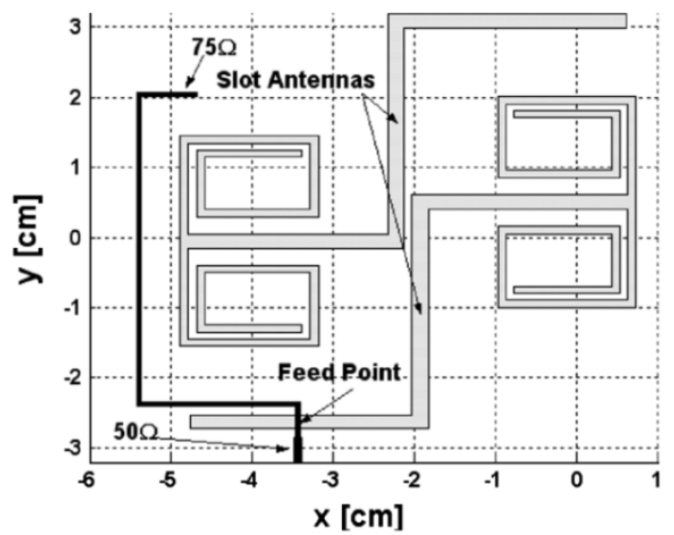

(b)

Fig. 1. Geometry of single- and double-element miniaturized slot antennas. (a) Single-element miniaturized slot antenna. (b) Double-element miniaturized slot antenna.

two miniaturized slot antennas are arranged so that they are parasitically coupled. Each antenna occupies an area of about $0.15 \lambda_{0} \times 0.13 \lambda_{0}$ [Fig. 1(a)] and achieves miniaturization by the virtue of a special topology described in detail in [6]. However, this antenna demonstrates a small BW (less than 1\%). A close examination of the antenna topology reveals that the slot-line trace of the antenna only covers about half of the rectangular printed-circuit board (PCB) area. Therefore another antenna, with the same geometry, can be placed in the remaining area without significantly increasing the overall PCB size. Placing two antennas in close proximity of each other creates strong coupling between the antennas which, if properly controlled, can be employed to increase the total antenna BW.

As seen in Fig. 1(b), only one of the two antennas is fed by a microstrip line. The other antenna is parasitically fed through capacitive coupling mostly at the elbow section. The coupling is a mixture of electric and magnetic couplings that counteract each other. At the elbow section, where the electric field is large, the slots are very close to each other; therefore, it is expected that the electric field coupling is the dominant coupling mechanism and the electric fields (magnetic currents) in both antennas will be in phase and thus the radiated far field is enhanced.

The two coupled antennas are designed to resonate at the same frequency, $f_{r 1}=f_{r 2}=f_{0}$, where $f_{0}$ is the center frequency and $f_{r 1}$ and $f_{r 2}$ are the resonant frequencies of the two antennas. In this case the $S_{11}$ spectral response of the coupled antenna will show two nulls, the separation of which is a function of the separation between the two antennas, $s$, and their overlap distance $d$. In order to quantify this null separation a coupling coefficient is defined as

$$
k_{t}=\frac{f_{u}^{2}-f_{l}^{2}}{f_{u}^{2}+f_{l}^{2}}
$$

where $f_{u}$ and $f_{l}$ are the frequencies of the upper and lower nulls in $S_{11}$. Hence $k_{t}$ can easily be adjusted by varying $d$ and $s$ [Fig. 1(b)], and decreases as $s$ is increased and $d$ is decreased. A full-wave electromagnetic simulation tool can be used to extract $k_{t}$ as a function of $d$ and $s$ in the design process. BW maximization is accomplished by choosing a coupling coefficient (by choosing $d$ and $s$ ) such that $S_{11}$ remains below $-10 \mathrm{~dB}$ over the entire frequency band. Here the resonant frequencies of both antennas are fixed at $f_{r 1}=f_{r 2}=850 \mathrm{MHz}$ and $k_{t}$ is used as the tuning parameter. However, it is also possible to change $f_{r 1}$ and $f_{r 2}$ slightly, in order to achieve a higher degree of control for tuning the response.

The input impedance of a microstrip-fed slot antenna, for a given slot width, depends on the location of the microstrip feed relative to one end of the slot and varies from zero at the short circuited end to a high resistance at the center. Therefore an off-center microstrip feed can be used to easily match a slot antenna to a wide range of desired input impedances. The optimum location of the feed line can be determined from the full-wave simulation. In the double antenna example the feed line consists of a $50 \Omega$ transmission line connected to an open-circuited $75 \Omega$ line crossing the slot [Fig. 1(b)]. The $75 \Omega$ line is extended by $0.33 \lambda_{m}$ beyond the strip-slot crossing to couple the maximum energy to the slot and also to compensate for the imaginary part of the input impedance. Using this $75 \Omega$ line as the feed, allows for compact and localized feed of the antenna and tuning the location of the transition from $50 \Omega$ to $75 \Omega$ provides another tuning parameter for obtaining a good match.

\section{B. Fabrication and Measurement}

A double-element antenna (DEA) and two different singleelement antennas (SEAs) (SEA 1 and SEA 2) were designed, fabricated, and measured. SEA 1 is the constitutive element of DEA and SEA 2 is an SEA with the same topology as SEA 1 [see Fig. 1(a)] but with the same area as the DEA. SEA 2 is used to compare the $\mathrm{BW}$ of the double resonant miniaturized antenna with that of the single-resonant miniaturized antenna with the same size. All antennas were simulated using IE3D [12] which is a full wave simulation software based on method of moments (MoM) and fabricated on a Rogers RO4350B substrate with thickness of $500 \mu \mathrm{m}$, a dielectric constant of $\epsilon_{r}=3.5$, and a loss tangent of $\tan (\delta)=0.003$ with a copper ground plane of $33.5 \times 23 \mathrm{~cm}^{2}$. The return losses of the SEAs as well as the DEA are presented in Fig. 2. SEA 1 shows a BW of $8 \mathrm{MHz}$ or nearly $0.9 \%$ and SEA 2 shows a BW of $11.7 \mathrm{MHz}$ or $1.31 \%$ whereas the BW of the DEA is $21.6 \mathrm{MHz}(2.54 \%)$ which indicates a factor of 1.94 increase over a SEA (SEA) with the 


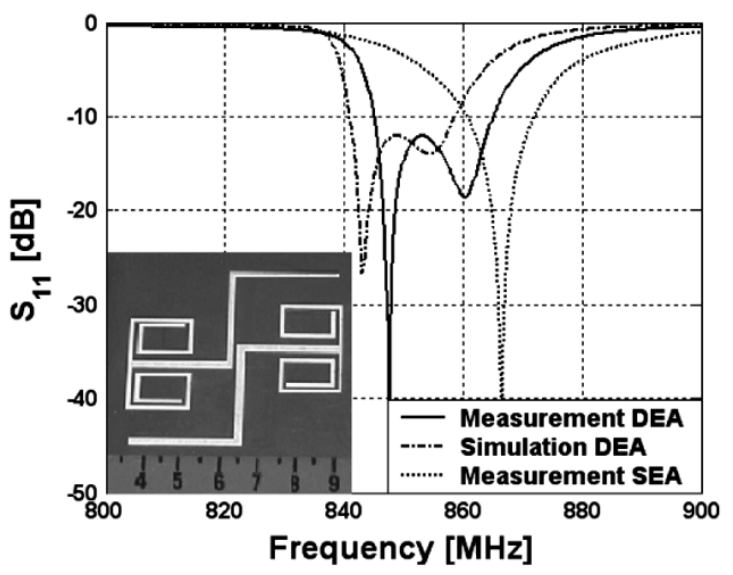

(a)

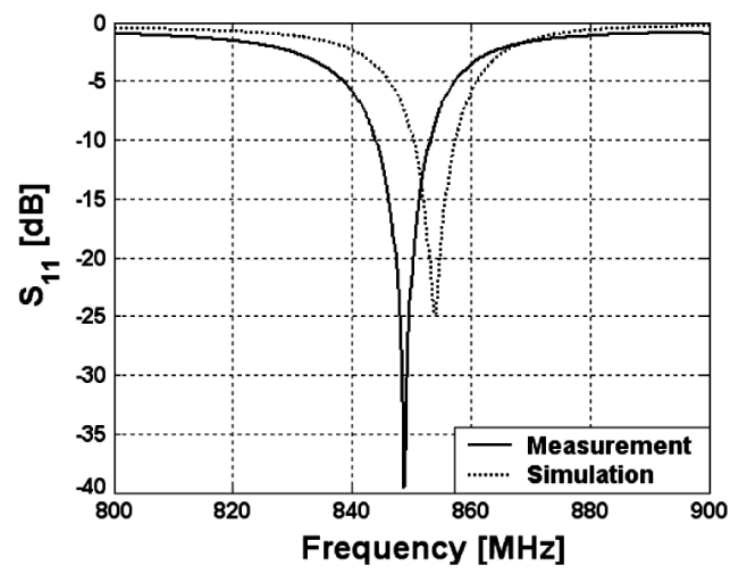

(b)

Fig. 2. Return losses of DEA and SEA 2: SEA with the same size as DEA. (a) Return losses of the DEA and SEA of the same size (SEA 2). (b) Return loss of the SEA that constitutes the DEA (SEA 1).

same area. Choosing a different substrate with different thickness and dielectric constant, can increase the overall BW of both antennas. However, it is also expected that the BW ratio of the DEA to the SEA remains the same. The overall size of the DEA is $0.165 \lambda_{0} \times 0.157 \lambda_{0}$ which shows a $25 \%$ increase in area when compared to the size of the SEA $1\left(0.133 \lambda_{0} \times 0.154 \lambda_{0}\right)$. The Q of each antenna has also been calculated using the method presented in [10] and compared with the fundamental limit on the $\mathrm{Q}$ of small antennas [4] in Table II. Demonstrably the quality factors of both SEAs are well above the minimum theoretical limit. Since Q is only defined for single resonant structures, no value for $\mathrm{Q}$ is reported for the DEA in Table II. In calculating the minimum $\mathrm{Q}$ for the slot antennas using the Chu limit, it is necessary to find the radius of the smallest sphere than encloses the antenna. At first, it may not be clear whether this sphere should only cover the aperture or, in addition to that, some portion of the ground plane too (because of the electric currents that exist in the ground plane). This becomes clear by applying the equivalence theorem to this problem, which shows that the magnetic currents responsible for radiation, exist only on the aperture and according the the derivation of the Chu limit, the smallest sphere that encloses these radiating magnetic currents should be used.

The gain of the double resonant antenna was measured at three different frequencies and is presented in Table I. Radiation patterns of the antenna were measured at $\mathrm{f}=848,852,860 \mathrm{MHz}$ and found to be similar to each other. Fig. 3 shows the co- and cross-polarized E- and H-plane radiation patterns at $\mathrm{f}=852 \mathrm{MHz}$. The $\mathrm{E}$ - and $\mathrm{H}$-plane radiation patterns of this antenna are expected to be dual of those of a short electric dipole. Fig. 3(a) shows the H-Plane radiation pattern which is similar to the E-Plane radiation pattern of an electric dipole with deeps instead of nulls at $\theta= \pm 90^{\circ}$. This can be attributed to the finiteness of the ground plane where some radiation comes from the electric currents on the antenna ground plane at the edges of the substrate. Fig. 3(b), however, does not show a uniform radiation pattern like the $\mathrm{H}$-Plane radiation of a short electric dipole. This is because of the $180^{\circ}$ difference in phase between the normal component of the electric fields at the top and bottom of the antenna ground plane. The H-Plane pattern is expected to have deep nulls at these angles; therefore, this does not significantly affect the
TABLE I

COMPARISON BETWEen the DEA AND ITS CONSTITUTIVE SEAs SHOWN IN FIG. 1

\begin{tabular}{c||c|c|c|c|c}
\hline \multicolumn{1}{c||}{ Type } & \multirow{2}{*}{$-10 \mathrm{~dB}$ BW } & \multicolumn{4}{|c}{ Gain } \\
\cline { 3 - 6 } & & $\mathrm{f}(\mathrm{MHz})$ & 848 & 852 & 860 \\
\hline Double Slot & $2.4 \%$ & $\mathrm{G}(\mathrm{dB})$ & 1.5 & 1.7 & 1.7 \\
\hline Single Slot 1 & $0.9 \%$ & \multicolumn{4}{|c}{$0.8 \mathrm{~dB}$} \\
\hline
\end{tabular}

TABLE II

Comparison Between Measured Q and the Minimum Attainable Q. * CAlCUlated USING THE Foster REACTANCE THEOREM [10]. ** CALCULATED USING THE CHU-MCLEAN FoRMULA FOR A Single-RESONANT ANTENNA [4]

\begin{tabular}{c||c|c|c}
\hline Type & Size & Measured Q* & Min $\mathrm{Q}^{* *}$ \\
\hline Single Element 1 & $0.133 \lambda_{0} \times 0.154 \lambda_{0}$ & 70.0 & 5.34 \\
\hline Single Element 2 & $0.165 \lambda_{0} \times 0.157 \lambda_{0}$ & 66.4 & 3.9 \\
\hline Double Element & $0.165 \lambda_{0} \times 0.157 \lambda_{0}$ & N/A & 3.9 \\
\hline
\end{tabular}

H-Plane pattern. Table I shows the radiation characteristics of the DEAs and SEAs. It is seen that the gain-BW product of the proposed double-antenna is significantly higher than that of the single antenna.

\section{IMPROVED ANTENNA MiNIATURIZATION USING DISTRIBUTED INDUCTIVE LOADING}

\section{A. Design Procedure}

A microstrip-fed slot antenna has the length of $\lambda_{g} / 2$, where $\lambda_{g}$ is the wavelength in the slot, at its first resonance. The electric current distribution can be modeled by the voltage distribution over a $\lambda / 2$ transmission line short circuited at both ends. The resonant length of a transmission line $\left(\lambda_{g}\right)$ can be made smaller if the inductance per unit length of the line is increased. This can be accomplished by inserting a number of series inductors in the transmission line. For slot-lines, insertion of series lumped elements is not possible. Besides, series lumped elements have a low $\mathrm{Q}$ which adversely affects antenna efficiency (gain). To realize a slot line with higher inductance per unit length, an array of distributed, short circuited, narrow slot-lines can be placed 


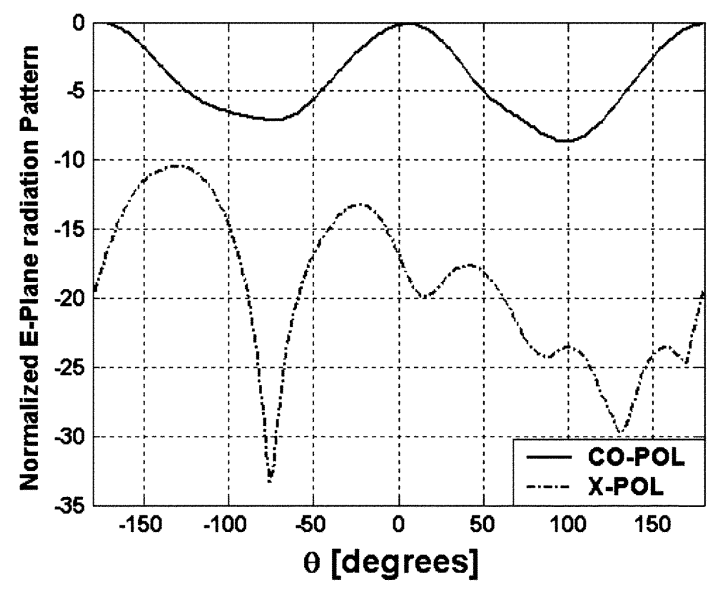

(a)

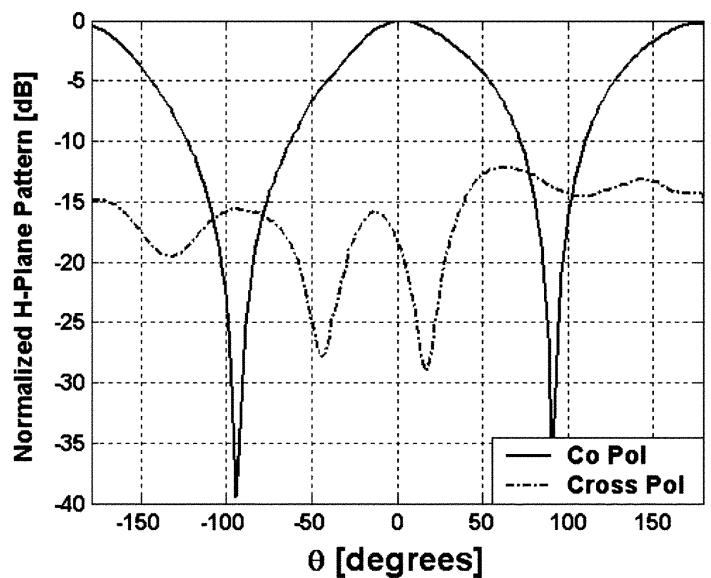

(b)

Fig. 3. Far field radiation patterns of the double-element miniaturized slot antenna at $852 \mathrm{MHz}$. (a) H-plane and (b) E-plane.

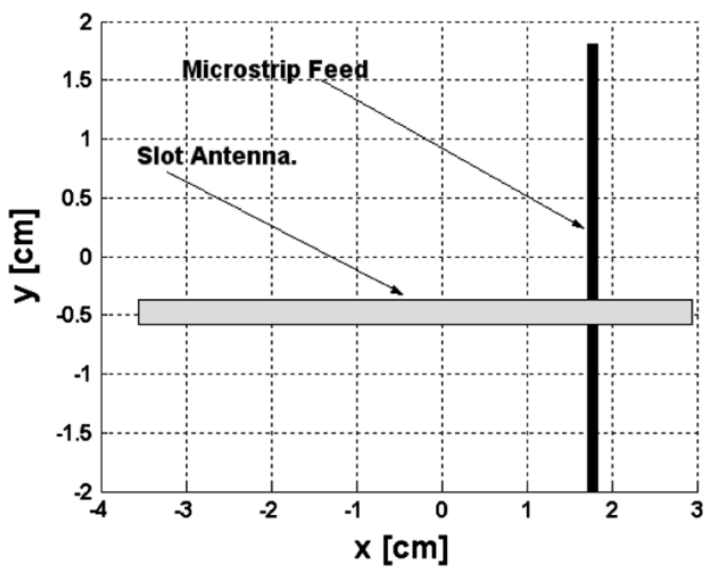

(a)

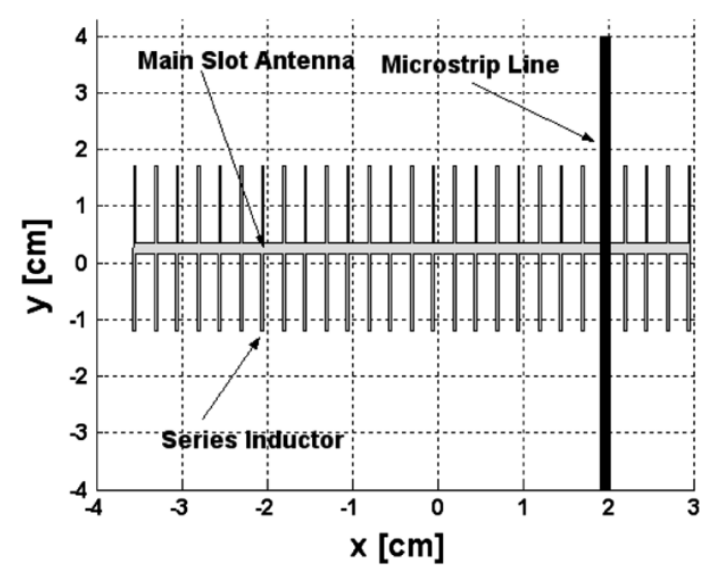

(b)

Fig. 4. Loaded and unloaded straight slot antennas. (a) Geometry of a microstrip-fed straight slot antenna. (b) Geometry of a microstrip-fed straight slot antenna loaded with an array of series inductive elements.

along the radiating segment of the slot antenna as shown in Fig. 4(b). The impedance of a short circuited slot line is obtained by

$$
Z_{S}=j Z_{0 s} \tan \left(\beta_{g} l\right)
$$

where $\beta_{g}$ is the propagation constant, $Z_{0 s}$ is the characteristic impedance, and $l$ is the length of the short circuited slot-line. The characteristic impedance of a slot-line is inversely proportional to its width [11] therefore by using wider series slots, more inductance can be obtained for a fixed length of short circuited transmission line. The best location to put series inductors in a slot is near its end where the amplitude of magnetic current is small. Putting them at the center of the slot where the magnetic current is at its maximum, strongly degrades radiation efficiency. It can easily be seen that by increasing the number and value of inductors, the length of transmission line necessary to satisfy the boundary conditions at both ends of the slot decreases.

The size reduction may also be explained by considering the electric current distribution in the conductor around the slot. There are two components of electric current in the ground plane of the slot, one that circulates around the slot and one that is

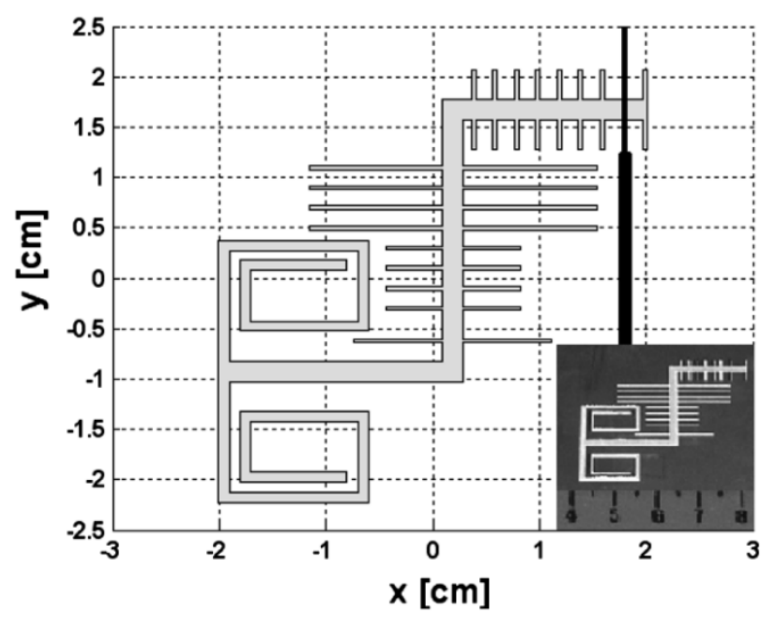

Fig. 5. Geometry of a miniaturized slot antenna loaded with series distributed inductors (slits).

perpendicular to it. The latter is described by the continuity of the electric current and displacement current at the slot discontinuity. Putting a discontinuity (a slit) normal to the circulating current path forces the current to circle around 


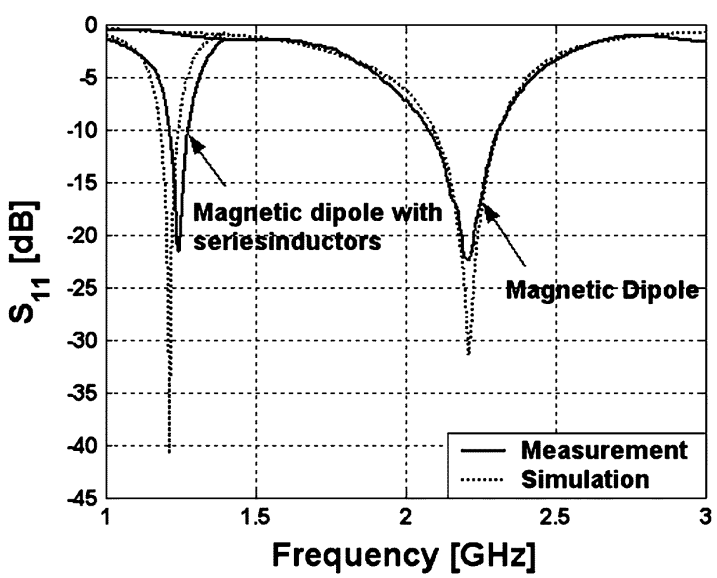

(a)

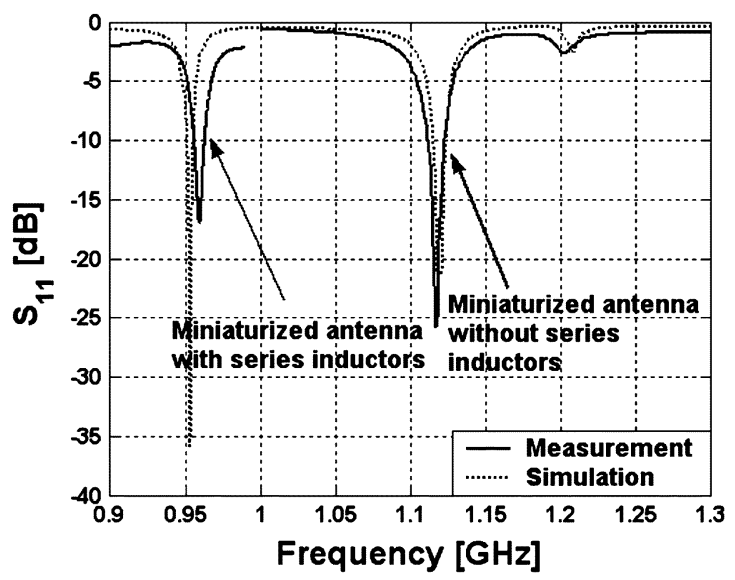

(b)

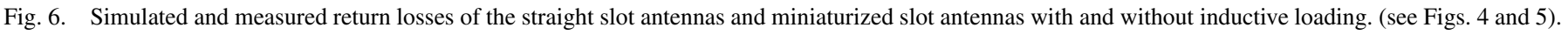
(a) Return losses of straight loaded and unloaded slot antennas. (b) Return losses of ordinary and loaded miniaturized slot antennas.

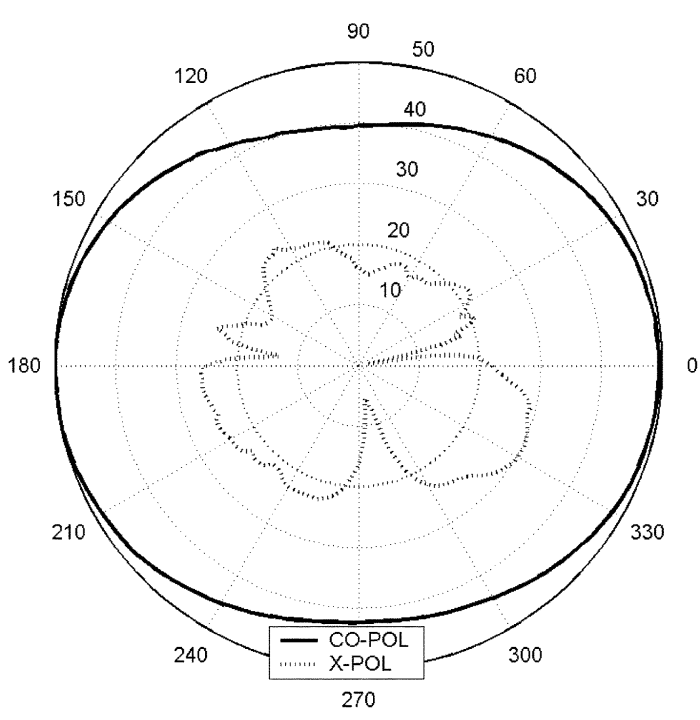

(a)

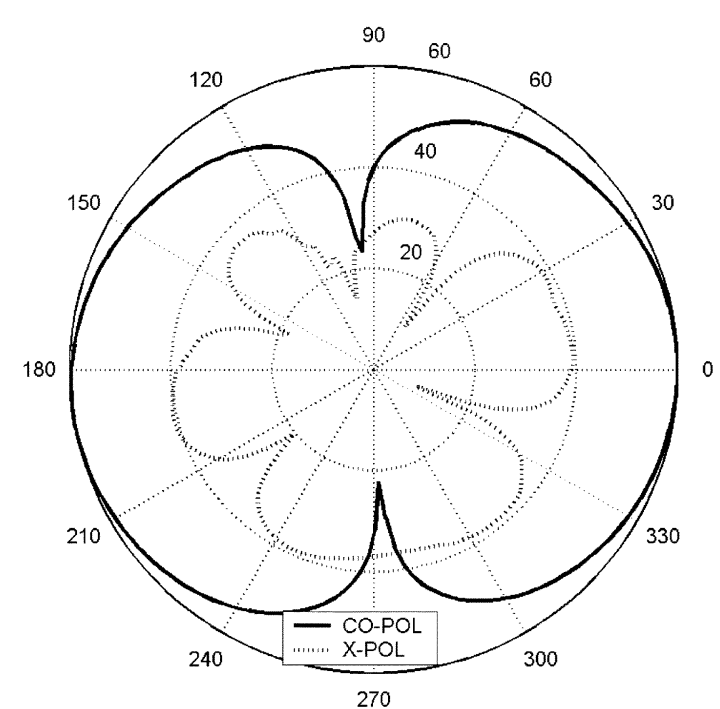

(b)

Fig. 7. Far field radiation patterns of loaded straight slot antenna shown in Fig. 4(b). (a) H-plane and (b) E-plane.

the discontinuity. Hence the electric current traverses a longer path length than the radiating slot length which in turn lowers the resonant frequency. Fig. 4(b) shows a slot antenna loaded with a number of narrow slits which act as an array of series inductors. These slits are designed to have a length smaller than $\lambda_{g} / 4$ and carry a magnetic current with a direction normal to that of the main radiator. Placing them only on one side of the radiating slot results in asymmetry in phase and amplitude of the current along the slot which could create problems in matching and worsen cross polarization. In order to circumvent this problem, two series slits are placed on the opposite sides of the main slot. These slits carry magnetic currents with equal amplitudes and opposite directions. Since the lengths of these narrow slits are small compared to the wavelength and since they are closely spaced, the radiated fields from the opposite slits cancel each other and they do not contribute to the radiated far field. Matching is performed by using an off-centered open circuited microstrip feed. The optimum location and length of the microstrip line are found by trial and error, using full wave simulations. For both straight slots (with and without series inductors) the lengths of the extended microstrip lines are found to be $\lambda_{m} / 4$, where $\lambda_{m}$ is the wavelength in the microstrip lines at their respective resonance frequencies.

Fig. 5 shows a miniaturized slot antenna (similar to the topology in [6]) loaded with series inductive slits to further reduce its resonant frequency. The antenna without the series inductors is already small, and adding series inductive elements further reduces the resonant frequency or equivalently the electrical dimensions of the antenna. Instead of using identical inductive elements along the radiating slot, differently sized inductive slits are used to cover most of the available area on the PCB in order to maintain the area occupied by the antenna. The antenna is matched to a microstrip transmission line in a manner similar to the straight slots described earlier. The feed line is composed of a $75 \Omega$ open-circuited microstrip line connected to a $50 \Omega$ feed line. In this case, the open circuited 


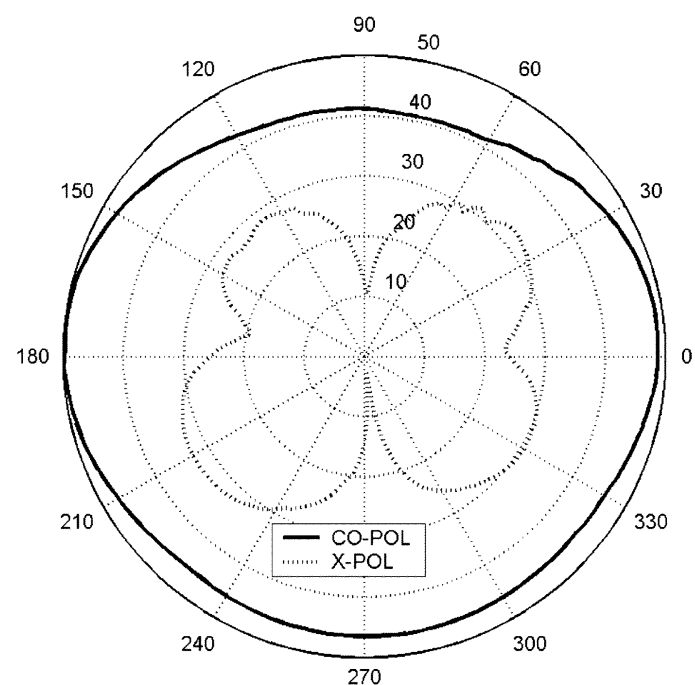

(a)

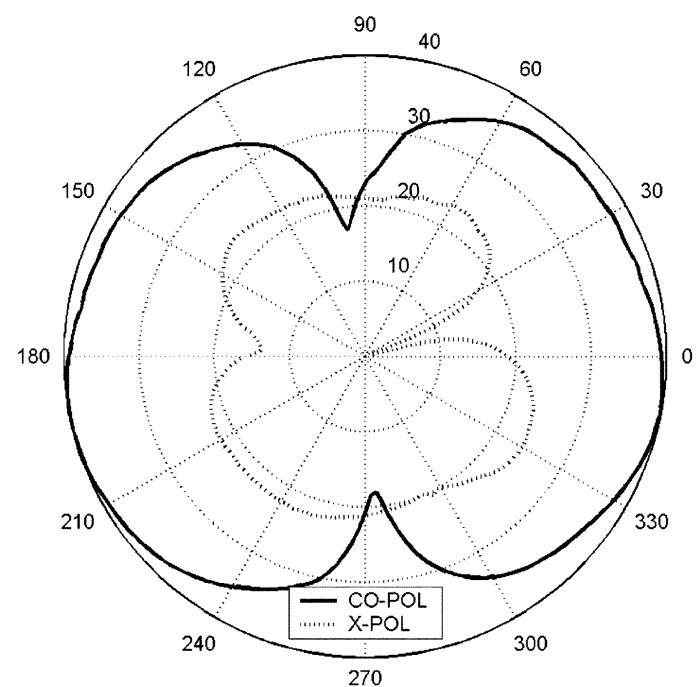

(b)

Fig. 8. Far field radiation patterns of loaded miniaturized slot antenna shown in Fig. 5. (a) H-plane and (b) E-plane.

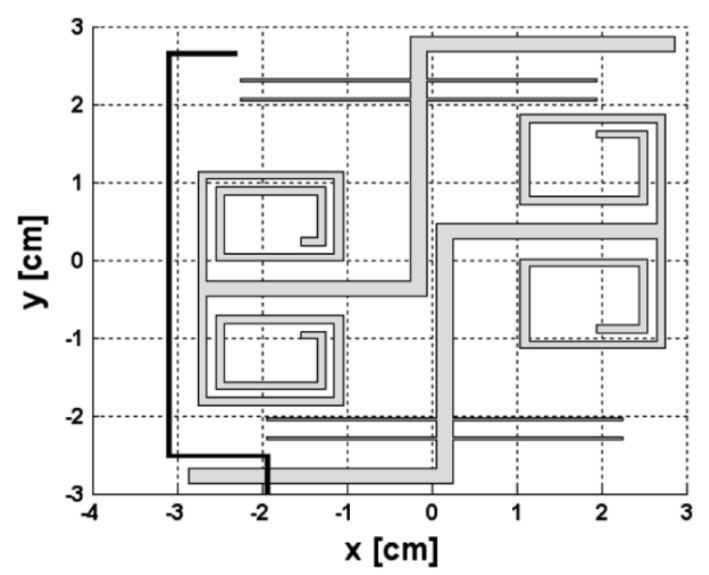

Fig. 9. Geometry of the dual band inductively loaded miniaturized slot antenna of Section IV.

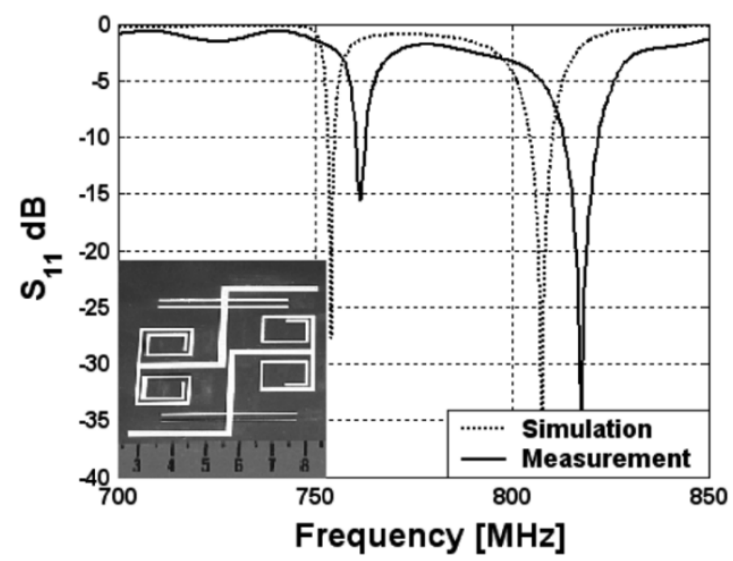

Fig. 10. Measured and simulated return losses of the miniaturized dual band slot antenna of Section IV.

microstrip line is extended beyond the slot-strip crossing by $0.3 \lambda_{m}$ and $0.26 \lambda_{m}$ for the miniaturized antenna and the loaded miniaturized antenna respectively.

\section{B. Fabrication and Measurement}

The straight slots with and without series inductors were simulated using IE3D and fabricated on a $500 \mu \mathrm{m}$ thick Rogers RO4350B substrate. Fig. 6(a) shows the simulated and measured return losses for the slot antennas with and without inductive loading. This figure shows the resonance frequency and $-10 \mathrm{~dB}$ BW of $2.2 \mathrm{GHz}$, and $235 \mathrm{MHz}(10.7 \%)$ for the straight slot. The loaded slot with the same length as that of the unloaded slot has a resonance frequency of $1.24 \mathrm{GHz}$ and a BW of $63 \mathrm{MHz}$ $(5 \%)$. This result indicates a $44 \%$ reduction in the resonant frequency and a similar reduction in the $\mathrm{BW}$, as expected. The overall size can still be reduced by using longer short circuited slits, if they could be designed in a compact fashion. The radiation patterns of the small slot antenna were measured in the anechoic chamber of the University of Michigan and are presented in Fig. 7. It is seen that the cross polarization components in the far field region in both E- and H-planes are negligible, thereby confirming the fact that the radiation from the magnetic currents in the inductive loadings with opposite directions cancel each other in the far field region.

The miniaturized loaded and unloaded slot antennas were also fabricated using RO4350B substrate. Fig. 6(b) shows the simulated and measured return losses of the loaded and unloaded miniaturized antennas. It is shown that, by inserting the series inductors, the resonant frequency of the antenna shifts down from 1116 to $959 \mathrm{MHz}$ (14\% reduction). In this design, the overall PCB size is unchanged. Fig. 8 shows the E- and H-plane co- and cross-polarized radiation patterns of the loaded miniaturized antenna. It is seen that the cross polarization level is negligible at broadside. The gains of the loaded and unloaded miniaturized slot antennas (antenna in Fig. 5) were also measured in the anechoic chamber using a standard log-periodic reference antenna and were found to be 0.8 and $0.7 \mathrm{~dB}$, respectively. Table III shows a comparison between $\mathrm{Q}$ of the miniaturized antennas presented in this section and the fundamental limit on Q of small antennas with the same size [1], [4]. It is observed that the $\mathrm{Q}$ of these antennas are well above the Chu limit. 
TABLE III

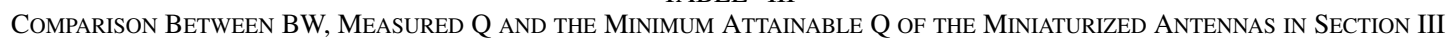

\begin{tabular}{c||c|c|c|c}
\hline Antenna Type & Size & $-10 \mathrm{~dB}$ BW & Measured Q & Min Q \\
\hline Min. Straight Slot & $0.260 \lambda_{0} \times 0.120 \lambda_{0}$ & $5 \%$ & 17.5 & 2.43 \\
\hline Min. S-shaped Slot & $0.15 \lambda_{0} \times 0.15 \lambda_{0}$ & $0.98 \%$ & 67.50 & 4.97 \\
\hline Loaded Min. S-Shaped Slot & $0.128 \lambda_{0} \times 0.128 \lambda_{0}$ & $0.73 \%$ & 98.4 & 7.21 \\
\hline
\end{tabular}

TABLE IV

Comparison Between BW, Measured Q and the Minimum Attainable Q of the Dual Band Miniaturized Antenna

\begin{tabular}{c||c|c|c|c}
\hline Resonance Frequency & Size & $-10 \mathrm{~dB}$ BW & Measured Q & Min Q \\
\hline $771.5 \mathrm{MHz}$ & $0.14 \lambda_{0} \times 0.15 \lambda_{0}$ & $0.4 \%$ & 200 & 5.25 \\
\hline $827.5 \mathrm{MHz}$ & $0.15 \lambda_{0} \times 0.16 \lambda_{0}$ & $1.2 \%$ & 104 & 4.44 \\
\hline
\end{tabular}

\section{DuAl Band Miniaturized Slot Antenna}

In this section the techniques introduced in the previous sections are used in the design of a dual band miniaturized slot antenna. The geometry of this antenna is shown in Fig. 9. The resonant frequencies of the slot antennas $\left(f_{r 1}\right.$ and $\left.f_{r 2}\right)$ and the value of the coupling coefficient $\left(k_{t}\right)$ are used as design parameters to achieve the desired response. Increasing the vertical displacement, $d$, and decreasing the horizontal separation, $s$, causes $k_{t}$ to increase or equivalently result in a larger separation between the two frequency bands. Small changes in the resonance lengths of the slots result in slight changes in $f_{r 1}$ and $f_{r 2}$ which can be used as a means of finetuning the response. Note, however, that resonant frequencies $f_{r 1}$, and $f_{r 2}$ should be close to each other so that coupling takes place. The separation between the two bands is limited by practical values of $k_{t}$. Large $k_{t}$ values cannot be obtained easily, since both electric and magnetic couplings are present and add destructively. In addition to this problem, matching the antenna at the two bands becomes increasingly difficult as the separation increases. A parameter $\delta f$ is defined as a measure of separation between the two frequency bands

$$
\delta f=\frac{\Delta f}{f_{0}}=\frac{f_{u}-f_{l}}{f_{0}}
$$

where $f_{0}$ is the center frequency. In practice by changing $k_{t}, f_{r 1}$, and $f_{r 2}$, values of $\delta f$ up to $10 \%$ can easily be obtained. This architecture is particularly useful for wireless applications that use two separate frequency bands (different bands for transmit and receive for example) that are close to each other but still cannot be covered with the available BW of these types of miniaturized antennas.

In order to achieve a higher miniaturization level for the given size, series inductive elements are also placed along slots to reduce the resonance frequencies of each element. Fig. 10 shows the simulated and measured return losses of this dual band antenna. The discrepancies between the simulated and measured results are due to the finiteness of the ground plane as described in [6]. The measured results indicate an $f_{l}=761.5 \mathrm{MHz}$ and $f_{u}=817.5 \mathrm{MHz}$ or equivalently a $\delta f=7.1 \%$. A good match at both bands is obtained by using an off center open-circuited microstrip feed where the microstrip line is extended by $7 \mathrm{~cm}$ over the slot-strip transition. Table IV shows a comparison between the antenna size, $-10 \mathrm{~dB} \mathrm{BW}$, measured $\mathrm{Q}$, and minimum attainable $\mathrm{Q}$ for the two bands. It is seen that the $\mathrm{Q}$ of both bands are well above the Chu limit. The overall size of the structure is $5.73 \mathrm{~cm} \times 5.94 \mathrm{~cm}$ or equivalently $0.145 \lambda_{0} \times 0.15 \lambda_{0}$ at the lowest frequency of operation. Radiation patterns of the antenna at the two bands are measured and found to be similar to those of the SEA topology (Fig. 8).

\section{CONCLUSION}

Two approaches are introduced for increasing the BW and reducing the size of miniaturized slot antennas. Placing two similar slot antennas in close proximity of each other creates a double resonant structure, the response of which is a function of relative spacing between the two antennas. The coupled miniaturized antenna can be designed to have a BW which is larger by $94 \%$ than the BW of a single resonant antenna with the same area or to behave as a dual band antenna.

For a fixed resonant frequency, adding series inductive elements to a slot antenna reduces its size. The size reduction is a function of number and values of the inserted inductive elements. Using series inductive elements does not adversely affect impedance matching and the cross polarization level. This technique is also used in combination with other miniaturization techniques to further decrease the size of the radiating structure. The technique is applied to a straight as well as a miniaturized slot antenna and for a given antenna size, significant reduction in resonant frequencies are observed.

Finally, both techniques are applied to the design of a miniaturized dual band antenna. Series inductors are used to reduce the resonant frequencies of each resonator. A large coupling coefficient is used to achieve a large separation between the two nulls in the $S_{11}$ response of the parasitically coupled antenna. The values of $k_{t}, f_{r 1}$, and $f_{r 2}$ are used as design parameters in order to obtain a miniaturized dual band slot antenna with relatively good simultaneous matching.

\section{ACKNOWLEDGMENT}

The authors would like to thank the anonymous reviewers for their constructive comments. 


\section{REFERENCES}

[1] L. J. Chu, "Physical limitations on omni-directional antennas," J. Appl. Phys., vol. 19, pp. 1163-1175, Dec. 1948.

[2] H. A. Wheeler, "Fundamental limitations of small antennas," in Proc. IRE., vol. 35, Dec. 1947, pp. 1479-1484

[3] R. C. Hansen, "Fundamental limitations in antennas," Proc. IEEE, vol. 69, pp. 170-182, Feb. 1981.

[4] J. S. McLean, "A re-examination of the fundamental limits on the radiation Q of electrically small antennas," IEEE Trans. Antennas Propagat., vol. 44, pp. 672-676, May 1996.

[5] H. K. Kan and R. B. Waterhouse, "Small square dual-spiral printed antennas," Electron. Lett., vol. 37, pp. 478-479, Apr. 2001.

[6] K. Sarabandi and R. Azadegan, "Design of an efficient miniaturized UHF planar antenna," in Proc. IEEE Int. Antennas Propagat. \& URSI Symp., Boston, MA, July 8-13, 2001.

[7] R. Azadegan and K. Sarabandi, "Miniaturized folded-slot: An approach to increase the bandwidth and efficiency of miniaturized slot antennas," in Proc. IEEE Int. Antennas Propagat. \& URSI Symp., San Antonio, TX, June 16-21, 2002.

[8] K. Sarabandi and H. Mosallaie, "Antenna miniaturization with enhanced bandwidth and radiation characteristics: A novel design utilizing periodic magneto-dielectric," in Proc. IEEE Int. Antennas Propagat. \& URSI Symp., San Antonio, TX, June 16-21, 2002.

[9] T. Ozdemir, P. Frantzis, K. Sabet, L. Katehi, K. Sarabandi, and J. Harvey, "Compact wireless antennas using a superstrate dielectric lens," in Proc. IEEE Trans. Antennas Propagat. \& URSI Symp., Salt Lake City, Utah, July 2000.

[10] W. Geyi, P. Jarmuszewski, and Y. Qi, "The foster reactance theorem for antennas and radiation Q," IEEE Trans. Antennas Propagat., vol. 48, pp. 401-408, Mar. 2000.

[11] J. J. Lee, "Slotline impedance," IEEE Trans. Microwave Theory and Techniques, vol. 39, pp. 666-672, Apr. 1991

[12] Electromagnetic Simulation and Optimization Software. IE3D

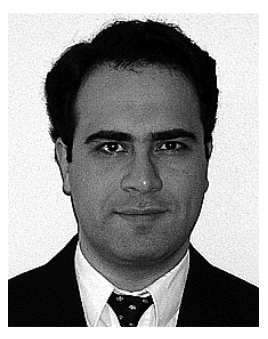

Nader Behdad (S'97) was born in Mashhad, Iran, in 1977. He received the Bachelor of Science degree from Sharif University of Technology, Tehran, Iran, and the Master of Science degree from the University of Michigan, Ann Arbor, in 2000 and 2003, respectively, where he is currently working toward the Ph.D. degree on bandwidth enhancement and miniaturization of printed antennas in the Department of Electrical Engineering and Computer Science.

From 2000 to 2001, he was with the Electronics Research Center, Sharif Unviersity of Technology, as an antenna design Engineer working on design of antennas for wireless local loop (WLL) systems. Since January 2002, he has been working as a Research Assistant in the Radiation Laboratory, University of Michigan.

Mr. Behdad is the recipient of the Best Student Paper Award in the Antenna Applications Symposium held in Monticelo, IL, in September 2003 and winner of the Second Prize in the student paper competition of the USNC/URSI National Radio Science Meeting, Boulder, CO, in January 2004.

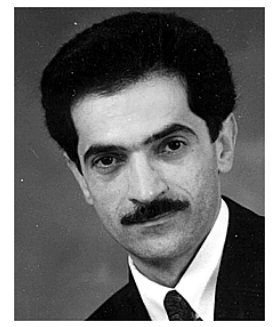

Kamal Sarabandi (S'87-M'90-SM'92-F'00) received the B.S. degree in electrical engineering from Sharif University of Technology, Tehran, Iran, in 1980, the M.S. degree in electrical engineering/mathematics, and the Ph.D. degree in electrical engineering from The University of Michigan, Ann Arbor, in 1986 and 1989, respectively.

$\mathrm{He}$ is Director of the Radiation Laboratory and a Professor in the Department of Electrical Engineering and Computer Science, The University of Michigan. He has 20 years of experience with wave propagation in random media, communication channel modeling, microwave sensors, and radar systems and is leading a large research group consisting of four research scientists, ten Ph.D. students, and two M.S. students. Over the past ten years he has graduated 15 Ph.D. students. He has served as the Principal Investigator on many projects sponsored by NASA, JPL, ARO, ONR, ARL, NSF, DARPA, and numerous industries. He has published many book chapters and more than 105 papers in refereed journals on electromagnetic scattering, random media modeling, wave propagation, antennas, microwave measurement techniques, radar calibration, inverse scattering problems, and microwave sensors. He has had more than 220 papers and invited presentations in national and international conferences and symposia on similar subjects. His research areas of interest include microwave and millimeter-wave radar remote sensing, electromagnetic wave propagation, and antenna miniaturization

Dr. Sarabandi is a Member of the International Scientific Radio Union (URSI) Commission F and of The Electromagnetic Academy. He received the Henry Russel Award from the Regent of The University of Michigan (the highest honor the University of Michigan bestows on a faculty member at the assistant or associate level). In 1999, he received a GAAC Distinguished Lecturer Award from the German Federal Ministry for Education, Science, and Technology. He also received a 1996 Teaching Excellence Award from the Department of Electrical Engineering and Computer Science, and the 2003/2004 College of Engineering Research Excellence Award, The University of Michigan. He is a Vice President of the IEEE Geoscience and Remote Sensing Society (GRSS), a past Chairman of the Awards Committee of the IEEE GRSS from 1998 to 2002, and a Member of the IEEE Technical Activities Board Awards Committee from 2000 to 2002. He is an Associate Editor of the IEEE TRANSACTIONS ON ANTENNAS AND PROPAgation and the IEEE SEnSORs Journal. He is listed in American Men \& Women of Science, Who's Who in America, and Who's Who in Electromagnetics. 